\title{
Thyroid-stimulating hormone within the normal range and risk of major adverse cardiovascular events in nonischemic dilated cardiomyopathy patients with severe left ventricular dysfunction
}

\author{
Xiaofei Li | Yan Yao @ | Zhaoran Chen | Siyang Fan | Wei Hua | Shu Zhang | \\ Xiaohan Fan (1)
}

State Key Laboratory of Cardiovascular Disease, Department of Cardiology, Fuwai Hospital, National Center for Cardiovascular Diseases, Chinese Academy of Medical Sciences and Peking Union Medical College, Beijing, China

\section{Correspondence}

Xiaohan Fan, MD, PhD, State Key Laboratory of Cardiovascular Disease, Department of Cardiology, Fuwai Hospital, National Center for Cardiovascular Diseases, Chinese Academy of Medical Sciences and Peking Union Medical College, No. 167, Beilishi Road, Xicheng District, Beijing 100037, China.

Email: fanxiaohan@fuwaihospital.org

Funding information

National Key R\&D Program of China , Grant/ Award Number: 2017 YFC1307800
Background: The association between thyroid-stimulating-hormone (TSH) and prognosis of nonischemic dilated cardiomyopathy (NIDCM) in patients with normal thyroid function remains unclear. Hypothesis: Our aim was to investigate the association between TSH and major adverse cardiovascular events in euthyroid NIDCM patients.

Methods: The original cohort consisted of 216 consecutive euthyroid NIDCM patients, with left ventricular ejection fraction (LVEF) $\leq 35 \%$, who were observed from 2010 to 2013 . Patients with persistent ventricular arrhythmia (VA) histories, amiodarone taken for VA prevention, or on heart transplant list within 1 year were excluded. A follow-up evaluation was performed, and VA events, heart failure (HF) exacerbation/heart transplant, cardiac death, or death from any cause were separately evaluated.

Results: A total of 184 patients were enrolled, and $97.8 \%$ (180/184) ultimately received follow-up evaluations. During the median 4.6-year follow-up, 24 VA events, 28 cardiac deaths, 30 all-cause deaths, $40 \mathrm{HF}$ exacerbations, and 11 heart transplant events occurred. Serum TSH levels showed good predictive efficacies for VA events (area under the curve $[A U C]=0.702,95 \%$ confidence interval [Cl]: 0.629-0.767), and the risk of VA events increased, according to serum TSH quarters, as determined by Kaplan-Meier analysis ( $2.2 \%$ vs $13.4 \%$ vs $21.0 \%$ vs $30.0 \%$, Q1-Q4, $P=0.011$ ). Multivariable Cox analysis showed that patients at the Q4 level of serum TSH (>2.67 mlU/L) suffered an increased risk of VA events, compared with those at the Q1 level of TSH (hazard ratio $[\mathrm{HR}]=15.88,95 \% \mathrm{Cl}: 2.01-65.15)$ or those at the other three quarters $(\mathrm{HR}=3.17,95 \% \mathrm{Cl}$ : 1.38-7.26). However, the Q4 TSH level was not associated with other adverse cardiac events. Conclusion: An association between TSH levels and the risk of VA events may exist in euthyroid NIDCM patients.

\section{KEYWORDS}

euthyroid, nonischemic dilated cardiomyopathy, thyroid-stimulating hormone, ventricular arrhythmias

\section{1 | INTRODUCTION}

Sudden cardiac death (SCD) is one of the most common etiologies for cardiac deaths in nonischemic dilated cardiomyopathy (NIDCM) patients with a reduced left ventricular ejection fraction (LVEF). The clinical application of implantable cardioverter-defibrillators (ICDs) has reduced the mortality of NIDCM patients with heart failure (HF) and was strongly recommended for NIDCM patients with a LVEF equal to or less than 35\%, based on the 2008 American College of Cardiology/ American Heart Association/Heart Rhythm Society guidelines for 
device-based therapy. ${ }^{1}$ However, in China, there were only $20 \%$ of patients with a Class I indication for ICD therapy who were available for an ICD implant, out of whom most were ICD secondary prevention candidates. ${ }^{2}$ A prophylactic ICD implantation for a primary SCD prevention is far from reaching the guideline recommendations, as an ICD implantation is still not covered by the national medical insurance of China. Beyond an ICD implantation, a nondevice therapy for ventricular arrhythmia (VA) prevention is still critically needed.

Thyroid hormone homeostasis is vital for the optimal functioning of the cardiovascular system. An abnormal thyroid function, whether it is overt or subclinical, was found to be related to deteriorations that include HF, cardiac deaths, and all-cause deaths in both ischemic and nonischemic cardiomyopathy patients. ${ }^{3,4}$ However, there has been a paucity of published data on the relationship between euthyroid function and major adverse cardiovascular events, especially for NIDCM patients with severe left ventricular (LV) dysfunction and SCD events. In the Rotterdam prospective population-based cohort study, ${ }^{5}$ higher levels of free thyroxine (fT4) in the euthyroid were related to an increased SCD risk. However, the patients enrolled in this study were all middle-aged and elderly members of the community population. Additionally, when considering the Sudden Cardiac Death in Heart Failure Trial (SCD-HeFT) study, ${ }^{6}$ an abnormal thyroid function (for both hypothyroid and hyperthyroid statuses) in HF patients was found to be associated with a higher mortality, while no differential SCD risks were observed.

Currently, no previous studies have published data on the association of the risks of major adverse cardiovascular events with a severely impaired LV function in euthyroid NIDCM patients. In order to fill this gap in the literature, we studied a prospective cohort, in order to analyze the association between euthyroid thyroid-stimulating-hormone (TSH) levels and major adverse cardiovascular events. Our hypothesis was that euthyroid NIDCM patients with an impaired LV function would be predisposed to different major adverse cardiovascular event risks, according to different TSH levels.

\section{2 | MATERIALS AND METHODS}

\section{1 | Participants}

We prospectively screened consecutive NIDCM patients with a LVEF $\leq 35 \%$, according to their echocardiography and normal thyroid function statuses, between December 2011 and December 2013, at the Fuwai Hospital in China. Additional inclusion criteria included patients with an optimal HF medication treatment for at least 3 months; an age below or equal to 65 years old; a New York Heart Association (NYHA) class II or III classification. A total of 32 patients were excluded from the cohort on the basis of the following criteria: a known coexisting obstructive coronary heart disease (including a history of myocardial infarctions or a history of acute coronary events); a malignant tumor; a scheduled heart transplant within 1 year; the use of amiodarone for VAs (patients with amiodarone treatment for atrial fibrillation, or atrial flutter patients, were not excluded) and an indication for the secondary prevention of SCD. The medication for the treatment of $\mathrm{HF}$ was based on the current guidelines-directed management ${ }^{7}$ and therapy, as well as the physicians' discretion. The dates of the patients' first evaluations were the time of the initial echocardiography examinations of each patient.

The ethics committee of the Cardiovascular Institute and Fuwai Hospital approved this study. All patients signed agreements to the use of their medical records for medical research, and all authors had full access to and took responsibility for the integrity of the data and had agreed to the manuscript as written.

\section{2 | Definition}

VA events were composited of a composite of SCD or appropriate ICD discharge, nonfatal ventricular fibrillation, or sustained ventricular tachycardia. ${ }^{8}$ SCD was defined as cardiac death occurred in a short time period ( 1 hour of symptom onset). ICD appropriate discharge refers to appropriate shock for sustained ventricular tachyarrhythmia. Nonsustained ventricular tachycardia (NSVT) was defined as three or more consecutive ventricular beats at a rate of more than 100 beats per minute which spontaneously terminates within 30 seconds. Frequent premature ventricular contraction (PVC) was defined as an average of 10 or more PVCs per hour while monitored. ${ }^{8}$ Hypertension and diabetes was defined according to 2009 European Society of Hypertension hypertension guideline ${ }^{9}$ and 2010 American Diabetes Association standards ${ }^{10}$ of medical care in diabetes.

Twelve-hour at least fasting blood samples were drawn and the serum levels of thyroid hormones were measured by radioimmunoassay (RIA) (Immulite 2000; Siemens, Munich, Germany) in the Nuclear Medicine Department of Fuwai Hospital. The reference intervals of thyroid hormones in our laboratory are as follows: TSH: 0.55 to $4.78 \mathrm{mIU} / \mathrm{L}$; free triiodothyronine (fT3): 1.79 to $4.09 \mathrm{pg} / \mathrm{mL}$; fT4: 0.8 to $1.88 \mathrm{ng} / \mathrm{dL}$; total triiodothyronine (TT3): 0.65 to $1.91 \mathrm{ng} / \mathrm{mL}$; total thyroxine (TT4): 4.29 to $12.47 \mu \mathrm{g} / \mathrm{dL}$. Patients with normal thyroid function or euthyroid was defined as TSH, fT3, and fT4 all within reference ranges, and no current levothyroxine or antithyroid drugs were used.

\subsection{Data collection, clinical follow-up, and endpoints}

Clinical data were obtained through reviewing of medical records. All baseline data was reviewed and analyzed by two separated physicians. Echocardiography was evaluated by two experienced echocardiography physicians, and LVEF was calculated using the modified Simpson biplane rule. All patients enrolled were followed up for every 3-month intervals via clinic or telephone visits (Figure 1). Major adverse cardiovascular events were collected by telephone or visiting follow-up and confirmed by reviewing medical records and household registrations records from public census department. For patients received ICD or cardiac resynchronization and implantable cardioverter-defibrillator (CRT-D) implantation, appropriated shocks were adjudicated by two experienced electrophysiological physicians blinded to all other clinical data. Events were classified as appropriate if they were a result of sustained ventricular tachyarrhythmia according to previous established criteria. ${ }^{11}$ Successful antitachycardia pacing therapy was not defined as one VA event in our study because most ventricular tachycardias 


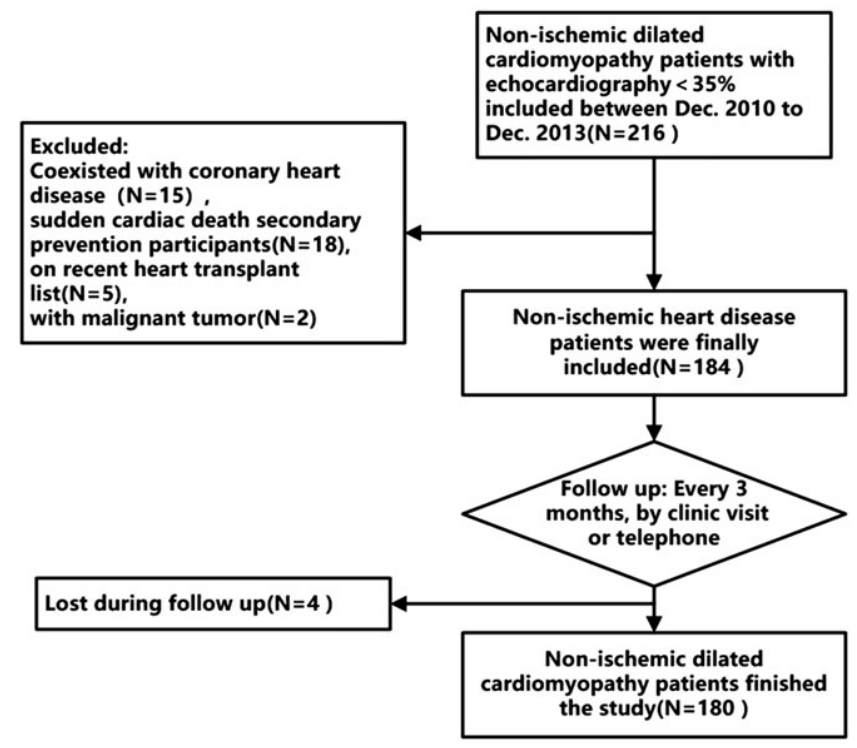

FIGURE 1 Flow-chat for the inclusion-exclusion of participants in the present study

that could be successfully treated by antitachycardia pacing are monomorphic and relatively nonfatal. Primary endpoint of the study was VA events, and the principal secondary endpoint was HF exacerbation hospitalization/heart transplant, cardiac death, or all-cause death, respectively. The follow-up duration was determined from the echocardiography examination date to the occurrence of an endpoint. If no endpoints occurred, data was collected until the last visit.

\section{4 | Statistical methods}

We performed statistical analyses with SPSS version 22.0 (SPSS, Inc., Chicago, Illinois) and GraphPad Prism 5 (Graphpad Software, Inc., San Diego, California). Continuous variables were presented as the mean $\pm S D$ and nominal data are presented as number and percentages. Baseline characteristics were compared among the groups using unpaired Student $t$ test or $\chi^{2}$ tests as appropriate. Receiver operating characteristics (ROC) curve was constructed to evaluate the predicting efficacy of TSH on VA events, HF exacerbation hospitalization/heart transplant, cardiac death, and allcause death risk. Kaplan-Meier (KM) survival curves among the groups stratified by TSH level were constructed and compared using the log-rank test. A Cox proportional hazards regression model was used to evaluate the role of TSH levels as a factor affecting major adverse cardiovascular events. $P$ value of $<0.05$ was considered significant.

\section{3 | RESULTS}

\subsection{Clinical outcomes}

During the median 4.6 years (range, 0.2-7.9 years) follow-up period, there were 12 patients who received an ICD or a CRT-D, and 14 patients received a cardiac resynchronization therapy pacemaker
(CRT-P) implantation. Fifty-five patients (30.6\%) suffered at least one of the clinical adverse events (including VA events, all-cause deaths, hospitalizations for HF exacerbation or heart transplants). VA events occurred in 24 primary prevention patients (13.3\%), out of whom 15 suffered an SCD, 5 suffered an appropriate ICD shock, and 5 survived from a sustained ventricular tachycardia or ventricular fibrillation. All-cause deaths occurred in 30 patients, and 28 of these deaths were classified as cardiac deaths. Hospitalizations for HF exacerbations occurred in 40 patients, and 11 patients received a heart transplant.

\subsection{Baseline characteristic of the study population}

Baseline characteristics of the NIDCM population are shown in Table 1. Patients were divided into four groups, according to the serum TSH level quarters (Q1-Q4). Patients in the TSH Q4 level were older, had more manifestations of atrial fibrillation/atrial flutter and NSVTs, had longer QRS durations, had larger left atriums and left ventricles and had taken more amiodarone, although no statistical significance was shown. There were no differences in serum fT3 and fT4 levels, NYHA grades, baseline blood pressures, and medications taken for chronic HF.

\section{3 | Relationship between TSH levels and major adverse cardiovascular events}

Major adverse cardiovascular events were evaluated by ROC and KM curve analyses, according to the TSH levels. Serum TSH levels were presented with a good prediction efficacy for VA events (area under the curve $[\mathrm{AUC}]=0.702,95 \%$ confidence interval $[\mathrm{Cl}]$ : $0.629-0.767, P<0.001$, Figure 2), while a poor efficacy was shown for the prediction of hospitalization for HF exacerbations/heart transplants (AUC $=0.529,95 \% \mathrm{Cl}$ : 0.453-0.603), cardiac deaths (AUC $=0.571, \quad 95 \% \quad \mathrm{Cl}: 0.496-0.645)$, and all-cause deaths (AUC $=0.546,95 \% \mathrm{Cl}: 0.471-0.621$ ). A KM curve analysis was used to assess the major adverse cardiovascular events at the median follow-up time point of 4.6 years. Patients with TSH levels in Q4 showed a distinctively higher cumulative risk for VA events than the other three quarters $(2.2 \%$ vs $13.4 \%$ vs $21.0 \%$ vs $30.0 \%$, log-rank $X^{2}=11.1, P=0.011$, Figure 3 ). For other adverse events, patients with TSH levels in Q4 showed a relatively higher risk of hospitalization for HF exacerbations/heart transplants than those with TSH levels for Q1 $(26.8 \%$ vs $20.2 \%$ vs $35.1 \%$ vs $27.4 \%$, log-rank $\left.X^{2}=2.43, P=0.49\right)$, cardiac deaths $(6.7 \%$ vs $15.9 \%$ vs $15.7 \%$ vs $21.4 \%$, log-rank $\left.X^{2}=3.89, P=0.273\right)$, and all-cause deaths $(9.1 \%$ vs $18.0 \%$ vs $15.7 \%$ vs $21.4 \%$, log-rank $X^{2}=3.28, P=0.35$ ), although no significant differences were found.

\subsection{TSH categories based on risk models for major adverse cardiovascular events}

Both univariate and multivariate Cox analyses was used to build the risk model. As shown in Table 2, compared to patients with TSH levels in Q1, those with TSH levels in Q4 showed a higher risk for VA events (hazard ratio [HR]: 13.20, 95\% Cl: 1.70-62.28), while no similar 
TABLE 1 Baseline characteristics of NIDCM patients according to TSH quarters

\begin{tabular}{|c|c|c|c|c|c|}
\hline \multirow[b]{2}{*}{ Variables } & \multicolumn{4}{|c|}{ Admission serum TSH level (mlU/L, $N=184$ ) } & \multirow[b]{2}{*}{$P$ value } \\
\hline & Q1 (0.55-1.16) & Q2 (1.17-1.84) & Q3 (1.85-2.67) & Q4 (2.68-4.78) & \\
\hline Age (years) & $35.1 \pm 10.1$ & $39.1 \pm 12.9$ & $35.7 \pm 12.9$ & $40.5 \pm 12.4$ & 0.104 \\
\hline $\mathrm{BMI}\left(\mathrm{kg} / \mathrm{m}^{2}\right)$ & $23.1 \pm 3.7$ & $25.7 \pm 4.5$ & $24.6 \pm 5.1$ & $25.2 \pm 4.5$ & 0.052 \\
\hline Hypertension (\%) & 17 (37.8) & $16(35.6)$ & $14(31.1)$ & $9(20.0)$ & 0.297 \\
\hline Diabetes (\%) & $6(13.3)$ & $9(20.0)$ & $3(6.7)$ & $3(6.7)$ & 0.149 \\
\hline Frequent PVC (\%) & 15 (33.3) & $11(24.4)$ & $18(40.0)$ & $18(40.0)$ & 0.351 \\
\hline NSVT (\%) & $2(4.4)$ & $2(4.4)$ & $4(8.8)$ & $6(13.4)$ & 0.314 \\
\hline AVB (II, \%) $)^{a}$ & $0(0)$ & $0(0)$ & $3(6.7)$ & $1(2.2)$ & 0.114 \\
\hline NYHA class III (\%) & $37(82.2)$ & $38(84.4)$ & $32(71.1)$ & $37(82.2)$ & 0.232 \\
\hline $\mathrm{SBP}(\mathrm{mm} \mathrm{Hg})$ & $108.2 \pm 17.0$ & $115.9 \pm 15.4$ & $113.2 \pm 18.0$ & $111.2 \pm 14.8$ & 0.159 \\
\hline QTc (ms) & $461.9 \pm 42.5$ & $455.7 \pm 41.4$ & $449.7 \pm 46.0$ & $454.8 \pm 53.6$ & 0.520 \\
\hline \multicolumn{6}{|l|}{ Lab tests } \\
\hline Hemoglobin (g/L) & $149.7 \pm 16.9$ & $153.9 \pm 13.5$ & $150.1 \pm 15.2$ & $149.1 \pm 19.4$ & 0.605 \\
\hline $\operatorname{Scr}(\mu \mathrm{mol} / \mathrm{L})$ & $89.8 \pm 38.8$ & $91.1 \pm 26.4$ & $89.6 \pm 20.7$ & $91.3 \pm 29.0$ & 0.827 \\
\hline NTproBNP (ng/L) & $2425.9 \pm 2159.0$ & $2320.3 \pm 2174.5$ & $1980.0 \pm 1321.3$ & $2215.9 \pm 2106.2$ & 0.776 \\
\hline Free T3 (pg/mL) & $2.88 \pm 0.47$ & $2.92 \pm 0.49$ & $3.00 \pm 0.45$ & $2.89 \pm 0.45$ & 0.599 \\
\hline Free T4 (ng/dL) & $1.31 \pm 0.23$ & $1.33 \pm 0.22$ & $1.38 \pm 0.24$ & $1.32 \pm 0.26$ & 0.459 \\
\hline \multicolumn{6}{|l|}{ Medication } \\
\hline ACEI/ARB (\%) & $32(71.1)$ & $39(86.7)$ & $40(88.9)$ & $38(84.4)$ & 0.125 \\
\hline LV diameter (mm) & $70.2 \pm 9.6$ & $68.0 \pm 9.1$ & $67.7 \pm 7.2$ & $71.5 \pm 8.4$ & 0.116 \\
\hline LVEF (\%) & $27.2 \pm 5.8$ & $29.7 \pm 5.7$ & $29.3 \pm 6.6$ & $28.5 \pm 5.9$ & 0.204 \\
\hline CRT-P (\%) & $1(2.2)$ & $4(8.7)$ & $4(8.7)$ & $5(10.9)$ & 0.721 \\
\hline ICD/CRT-D (\%) & $1(0.0)$ & $3(2.9)$ & $4(2.9)$ & $4(0)$ & 0.817 \\
\hline
\end{tabular}

Abbreviations: $\mathrm{ACEI} / \mathrm{ARB}$, angiotensin converting enzyme inhibitors or angiotensin II receptor antagonists; AF/AFL, atrial fibrillation or atrial flutter; AVB, atrioventricular block; BMI, body mass index; DBP, diastolic blood pressure; CRT-P, cardiac resynchronization therapy pacemaker; DBP, diastolic blood pressure; Echo, echocardiography; ICD/CRT-D, implantable cardioverter-defibrillator/cardioverter-defibrillator; LA, left atrium; LV, left ventricle; LVEF, left ventricular ejection fraction; NIDCM, nonischemic dilated cardiomyopathy; NSVT, nonsustained ventricular tachycardia; NT-proBNP, N-terminal pro brain natriuretic peptide; NYHA, New York heart association; PVC, premature ventricular contraction; QTc, heart rate-adjusted QT; SBP, systolic blood pressure; Scr, serum creatinine; T3, triiodothyronine; T4, thyroxine; TSH, thyroid stimulating hormone.

${ }^{a}$ AVB: second degree or more severe atrioventricular block.

prediction value was found for the other major adverse cardiovascular events. When age, sex, NSVT history, NYHA class, and LVEF were adjusted, patients with TSH levels in Q4 suffered a greater number of VA events (HR 15.88, 95\% Cl: 2.01-65.15) than those with TSH levels in Q1. After adjustments for age, sex, atrial fibrillation or atrial flutter (AF/AFL) history, NYHA class, and LVEF, patients with TSH levels were not associated with increased hospitalizations for HF exacerbations/heart transplants, cardiac deaths, or all-cause death risks (Table 2). Furthermore, the AF/AFL history and low LVEF were independent risk factors for cardiac deaths (AF/AFL: $H R=3.84,95 \% \mathrm{Cl}=$ 1.69-8.75, $P=0.001 ; \quad$ LVEF: $\quad H R=0.92, \quad 95 \% \quad C l=0.86-0.98$,
$P=0.013)$ and all-cause deaths (AF/AFL: $H R=4.03,95 \% \mathrm{Cl}=$ 1.82-8.94, $\quad P=0.001 ; \quad$ LVEF: $\quad H R=0.91, \quad 95 \% \quad C l=0.86-0.97$, $P=0.008$ ) in NIDCM patients with severe LV dysfunction (Table S1, Supporting Information).

The risk of adverse cardiovascular events in TSH Q4 patients was also compared with all other quarters of TSH (Figure S1). Patients in TSH Q4 were shown to have a higher risk of VA events than other patients $(\mathrm{HR}=3.17,95 \% \mathrm{Cl}=1.38-7.26, P=0.006)$. Additionally, for other risks of major adverse events, no significant difference was found in TSH levels of Q4 patients. Furthermore, for the risks of cardiac deaths and all-cause deaths, atrial fibrillation, or atrial flutter 
(A)

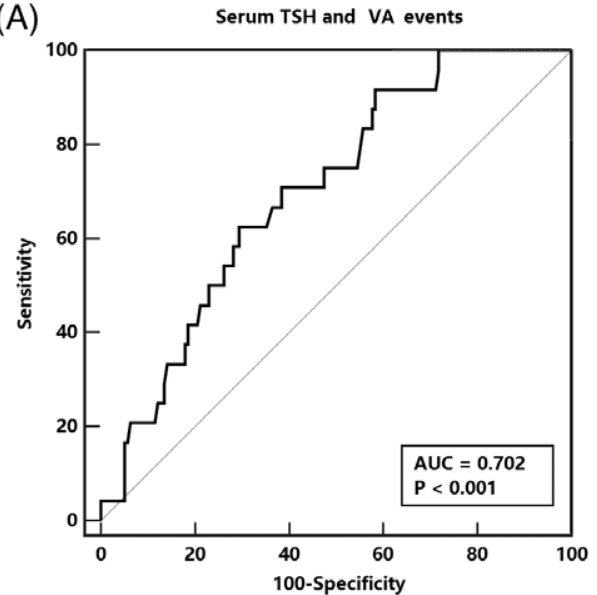

(C)

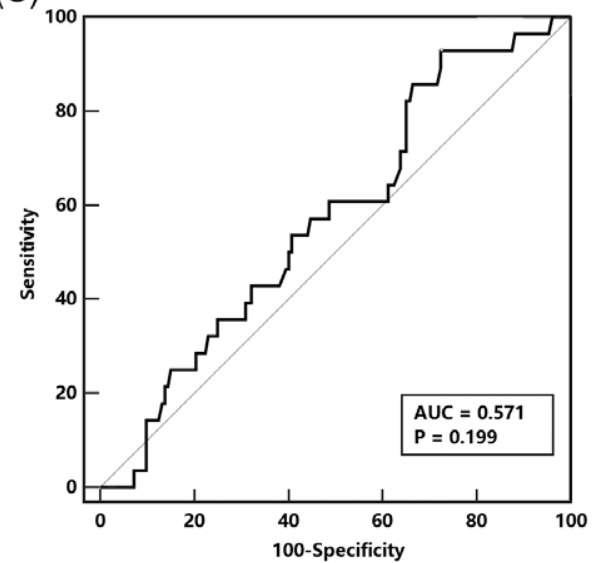

(B)

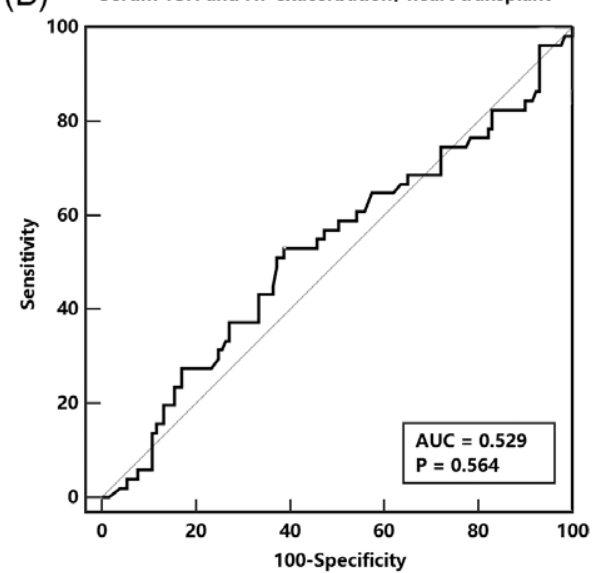

(D)

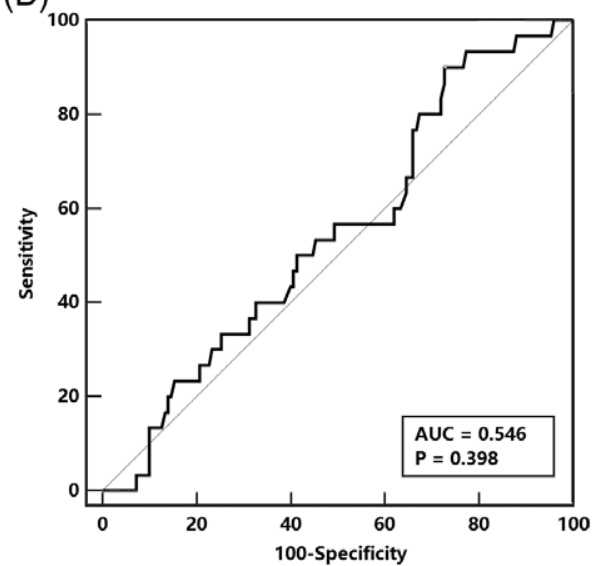

FIGURE 2 ROC curve for serum TSH and major adverse cardiovascular events. HF, heart failure; ROC, receiver operating characteristics; TSH, thyroid stimulating hormone; VA, ventricular arrhythmia

history, as well as low LVEF, were also presented as independent risk factors (Table S2).

\section{4 | DISCUSSION}

In the current study, TSH levels in Q4 (>2.67 mIU/L) were associated with increased relative and absolute risks of VA events in a NIDCM SCD primary prevention population, with thyroid function within the normal range. Other risks of adverse outcomes, including hospitalizations for HF exacerbations/heart transplants and risks of cardiac death, and all-cause death, did not vary between the different TSH quarters. This is the first study addressing the association between TSH and the risk of major adverse cardiovascular events in an SCD primary prevention population with euthyroid statuses.

\section{1 | Thyroid status and adverse cardiac events}

Thyroid hormones are important regulators of the entire cardiovascular system. Directly, a beta-adrenergic positive chronotropic effect, which is regulated by thyroid hormones, can lead to a hyperdynamic state, which can increase the cardiac preload and ventricular filling pressure, thus resulting in the cardiac tachycardia. ${ }^{12}$ Furthermore, abnormal thyroid hormone levels (in both hyperthyroid and hypothyroid statuses) have been shown to lead to atrial fibrillation ${ }^{13}$ and QT prolongation, ${ }^{14,15}$ which is closely related to SCD events. ${ }^{16,17}$ Several earlier studies have evaluated the relationship between thyroid function and VA risks. However, these studies were focused on the hypothyroid/hyperthyroid statuses (both clinical or subclinical), and the enrolled population ranged from a general community population ${ }^{5,18}$ and $\mathrm{HF}$ patients ${ }^{6,19}$ to diabetic hemodialysis individuals, ${ }^{20}$ for whom VA risks were not significantly high when compared to patients with an indication for ICD primary prevention. In the SCD-HeFT trial ${ }^{6}$ and Controlled Rosuvastatin Multinational Study in Heart Failure trial, ${ }^{19}$ the only two studies that have evaluated thyroid function in an ICD primary implantation population, abnormal thyroid hormone levels (both hypo- or hyperthyroid statuses) were related with adverse cardiac events, with the SCD or ICD therapy statuses not being fully evaluated. In the current study, the traditional SCD-related risk factors (including LVEF, NYHA class, atrial fibrillation, age, and NSVT) and sex were adjusted, and VA events were found to be more prevalent in TSH Q4 euthyroid patients. We suspect that the risk of VA events may be lower when TSH is at a lower level, or within the "normal range".

Subclinical hypothyroidism-related bradycardia, impaired systolic function, increased systemic vascular resistance and endothelial 


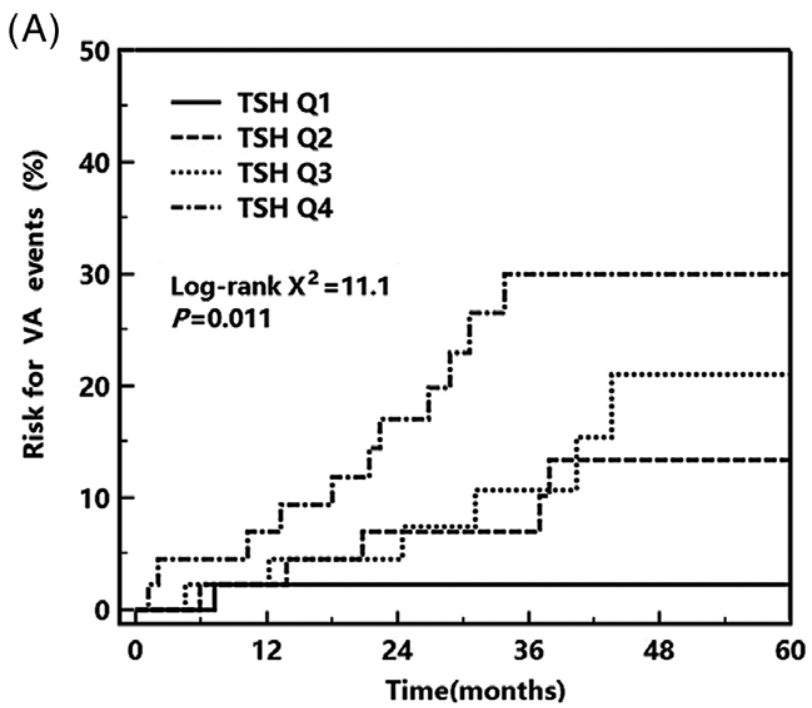

$\begin{array}{llllll}\text { Number at risk } & & & & & \\ \text { TSH Q1 } 45 & 43 & 39 & 23 & 11 & 4 \\ \text { TSH Q2 } 45 & 43 & 37 & 30 & 14 & 4 \\ \text { TSH Q3 } 45 & 43 & 34 & 25 & 9 & 1 \\ \text { TSH Q4 } 45 & 39 & 32 & 20 & 2 & 1\end{array}$

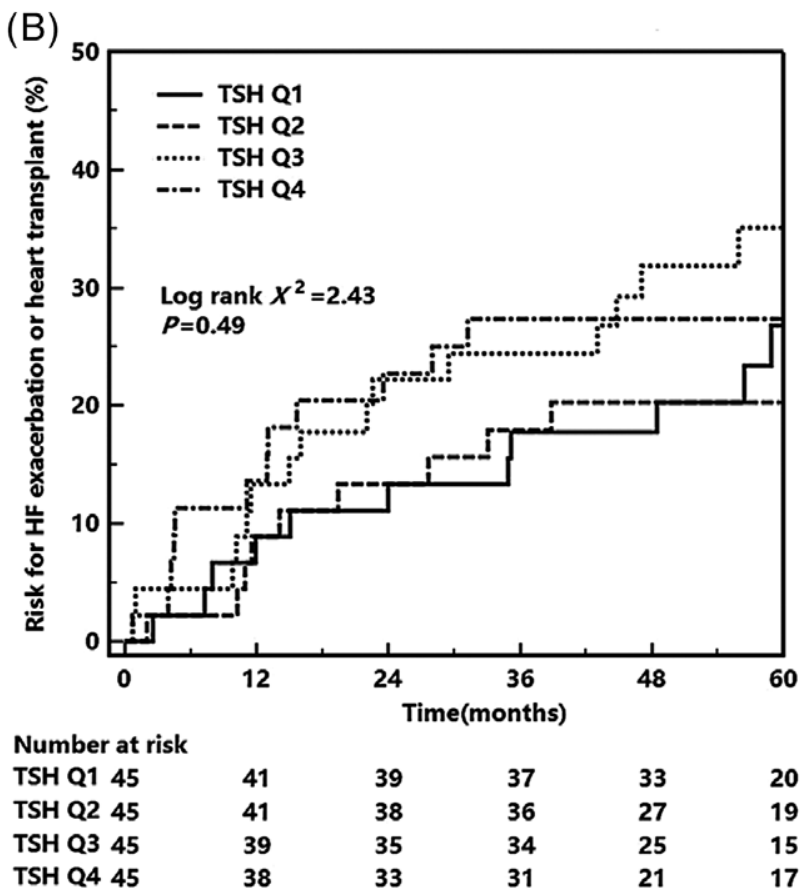

(C)
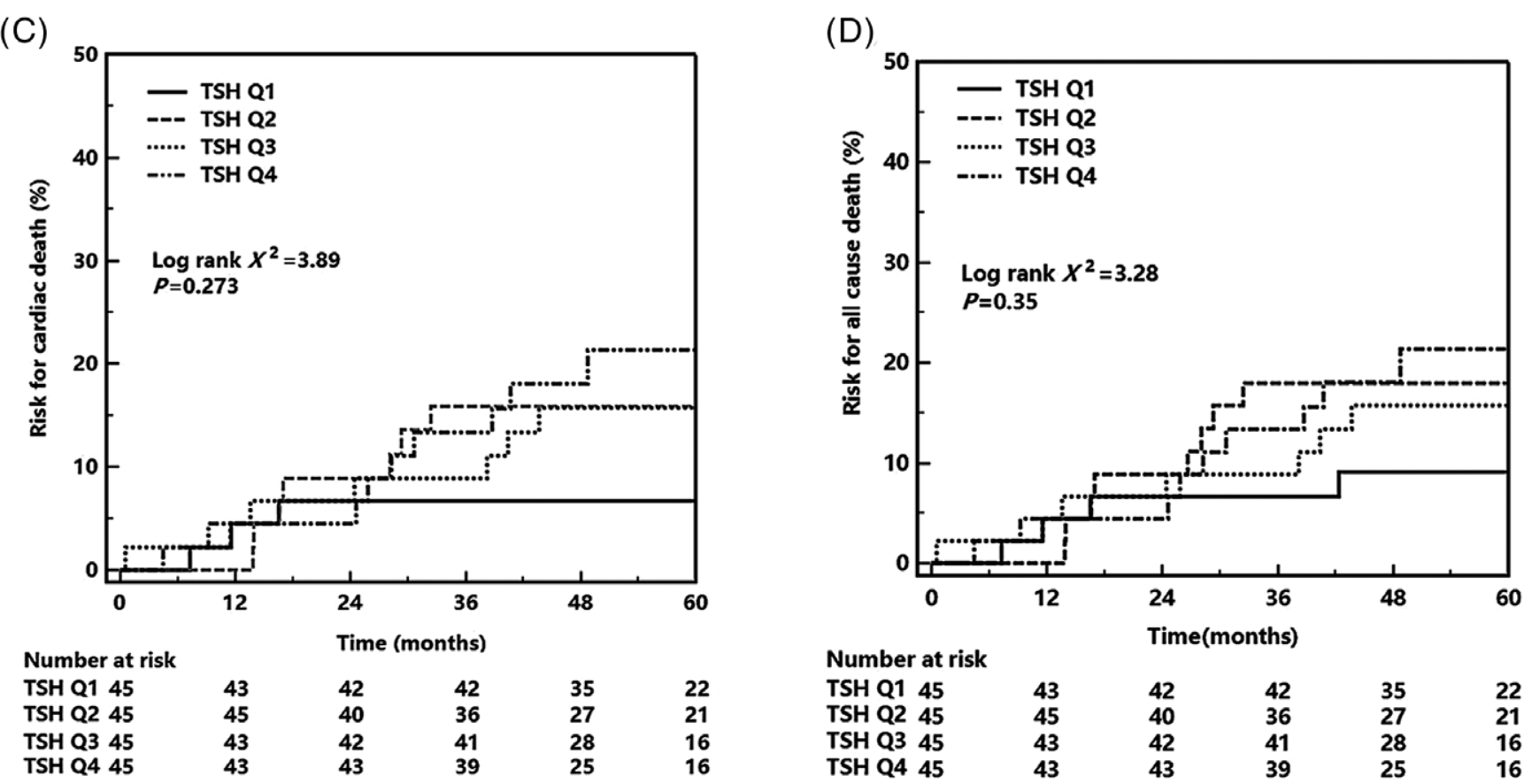

FIGURE 3 KM analysis for serum TSH and major adverse cardiovascular events. KM, Kaplan-Meier; TSH, thyroid stimulating hormone

dysfunction ${ }^{12}$ may play important roles in the progression of major adverse cardiovascular events. The SCD-HeFT study ${ }^{6}$ had found that a subclinical hypothyroid is associated with increased mortality in HF patients. In the CORONA trial ${ }^{19}$ and the recently published Seo's study, ${ }^{21}$ subclinical hypothyroidism was associated with an increased risk of composite cardiac death, HF hospitalization and all-cause death, although this relationship was eliminated when other known predictors were adjusted. In Wang's ${ }^{22} \mathrm{TSH}$ and dilated cardiomyopathy cohort study, subclinical hypothyroidism was also found to be associated with increased mortality in a Chinese dilated cardiomyopathy population. However, in this study, all-cause deaths, cardiac deaths, and HF rehospitalization or heart transplant risks were similar between the different TSH quarters, which appears to differ from the studies mentioned previously. These differences might be caused by the following reasons. First, patients with different TSH quarters shared similar baseline HF severities, which can be reflected from the baseline brain natriuretic peptide (BNP) levels and LVEF by an echocardiography. Second, the predictive value of TSH for adverse cardiac events, such as HF deterioration, may be limited to specific populations, such as the elderly ${ }^{23}$ or black populations. ${ }^{24}$ However, most of the patients included in this study were young and middle-aged (mean age: 37.6-years-old), which was much lower than the average age of the patients in the SCD-HeFT study (mean age: 59.5 -years-old), ${ }^{6}$ as well as the patients in the CORONA trial (mean age: 72.6 -years-old) ${ }^{19}$ 
TABLE 2 Major adverse cardiovascular events according to TSH quarters based on cox analysis

\begin{tabular}{|c|c|c|c|c|}
\hline \multirow[b]{2}{*}{ Outcome } & \multicolumn{4}{|c|}{ Baseline TSH (mIU/L) categories and HR $(95 \% \mathrm{Cl})$} \\
\hline & Q1 (0.55-1.16) & Q2 (1.17-1.84) & Q3 (1.85-2.67) & Q4 (2.68-4.78) \\
\hline \multicolumn{5}{|l|}{ Crude model } \\
\hline HF exacerbation or $\mathrm{HTx}$ & Ref. (1.0) & $0.70(0.30-1.65)$ & $1.22(0.57-2.59)$ & $1.20(0.56-2.57)$ \\
\hline Cardiac death & Ref. (1.0) & $3.21(0.87-11.84)$ & $2.44(0.63-9.45)$ & $3.20(0.87-11.85)$ \\
\hline All-cause death & Ref. (1.0) & $2.68(0.84-8.55)$ & $1.83(0.54-6.26)$ & $2.41(0.74-7.83)$ \\
\hline VA events & Ref. (1.0) & $6.95(0.82-39.10)$ & $7.78(0.93-45.38)$ & $15.88(2.01-65.15) *$ \\
\hline HF exacerbation or $\mathrm{HTx}$ & Ref. (1.0) & $0.78(0.33-1.87)$ & $1.47(0.68-3.20)$ & $1.35(0.61-2.97)$ \\
\hline Cardiac death & Ref. (1.0) & $3.80(0.98-14.59)$ & $2.46(0.63-9.63)$ & $3.37(0.90-12.65)$ \\
\hline All-cause death & Ref. (1.0) & $3.17(0.95-10.54)$ & $1.85(0.54-6.39)$ & $2.50(0.76-8.25)$ \\
\hline
\end{tabular}

Abbreviations: $\mathrm{Cl}$, confidence interval; HF, heart failure; HTx, heart transplant; NYHA, New York Heart Association; SCD, sudden cardiac death; TSH, thyroid-stimulating-hormone; $\mathrm{VA}$, ventricular arrhythmia.

a VA events were adjusted for age, sex, nonsustained ventricular tachycardia, NYHA class, and left ventricular ejection fraction; other adverse events were adjusted for age, sex, atrial fibrillation or atrial flutter, NYHA class, and left ventricular ejection fraction $* P<0.05$

population. In addition, all of the people included in this study were members of the Asian population. Third, all of the enrolled patients had euthyroid statuses, which was different from the previous studies, which involved populations with different abnormalities of thyroid function status.

In addition to our study, the "normal range" of thyroid function has been challenged in many studies. In the Rotterdam study, 5 enrolled community patients over 45-years-old, who had higher free thyroxine levels within the normal range, had predicted 10-year SCD risks. In the Interdisciplinary Network Heart Failure study, ${ }^{25}$ the euthyroid sick syndrome (involving low circulating triiodothyronine [T3] levels in patients with normal, or slightly decreased, TSH and T4 concentrations and viewed as an adaptive response to serious clinical impairments, rather than as a genuine thyroid disease) was observed as a predictor of a poor prognosis of HF patients. In Christine's metaanalysis $^{13}$ that examined 279000 enrolled individuals, higher free thyroxine levels in euthyroid individuals were reported as being independent risk factors for the incidence of atrial fibrillations. This is likely because the normal ranges for thyroid hormones were based on the distributions in apparently healthy populations, which may not correctly mirror the real thyroid status in specific situations, such as in the case of a HF state. Chaker ${ }^{5,26}$ had proposed the theory that the optimal health range for thyroid function should be redefined based on the risk of cardiovascular disease. In his study, individuals with fT4 levels over the 90th percentile and within the normal range suffered a higher risk of cardiovascular death. These studies may reveal that, for patients with specific conditions (eg, in this study, NIDCM patients with a severely impaired LV function), the "normal" range of thyroid function should be redefined by the SCD event, or by cardiac death risk.

\subsection{Amiodarone and thyroid function in NIDCM patients}

Amiodarone, especially at higher doses (> $400 \mathrm{mg} /$ day) in which the large iodine content of amiodarone increases plasma inorganic iodide by 40 -fold per 24 hours, may increase serum TSH levels. ${ }^{27}$ Usually, TSH levels may initially increase, then decrease slightly with small range fluctuations, after which a relatively higher level is maintained, which mainly results in a relatively hypothyroid status. ${ }^{6}$ Meanwhile, for ICD candidates, amiodarone was more easily prescribed for patients with a high risk for VA, and amiodarone-related TSH increases may attenuate the predictive value of TSH levels. However, in this study, only 16 patients took a small dose ( $200 \mathrm{mg} /$ day) of amiodarone for a sinus rhythm control (and only for AF/AFL patients). Additionally, for TSH Q3 and Q4 patients, a similar amiodarone administration rate did not result in a similar VA risk. Furthermore, in the multivariable analysis, patients with an atrial fibrillation or an atrial flutter were also adjusted, and patients with TSH levels in Q4 still suffered a higher VA risk.

LVEF is the main criteria for ICD implantation in NIDCM patients under the current VA management guidelines. ${ }^{28}$ Previous studies ${ }^{29,30}$ have reported that a lower LVEF (LVEF $<25 \%$ or $30 \%$ ) may represent a higher SCD risk. However, this prediction efficacy was more prevalent for ischemic heart disease with an LV dysfunction, instead of NIDCM. ${ }^{28}$ NSVT is a relatively common finding in patients with structurally abnormal hearts. However, only NSVT in ischemic patients can predict further mortality. ${ }^{31}$ For a patient with NIDCM, the prognostic significance of NSVT is uncertain, and no studies have provided precise guidance for treatment in this group of patients. ${ }^{32}$ In this study, LVEF and NSVT were not independent risk factors for those NIDCM patients with a severe dysfunction of the LV in predicting SCD events.

SCD events always develop immediately, and time is limited in starting cardiopulmonary resuscitation. Even for SCD primary prevention patients with a class I recommendation, ICD cannot always be implanted, according to the patient's motivation and economic considerations, among others. Therefore, the identification of modifiable risk factors is crucial for the identification of certain populations at risk and is also crucial for screening and prevention. Based on our study, NIDCM patients with a high VA risk (such as an ICD primary prevention population) had TSH levels maintained within a lower normal 
range, which may decrease the VA risk. It is predicted that, for ICD implantation patients, TSH levels in lower, normal range may also decrease unnecessary ICD discharges, although further studies are still needed to be performed. However, in our study, no specific conclusions on the benefits of thyroid medication can be drawn. A previous randomized, crossover, placebo-control study ${ }^{33}$ had shown that an oral T3 treatment may not be beneficial in patients with a moderate LV dysfunction (a mean ejection fraction of 43\%). Additionally, for severe LV dysfunction patients, large multicenter trials that document a definite role of thyroid hormone treatment are still lacking.

Despite strong evidence supporting our results, this study had several limitations. First, the sample size was relatively small, and the power for analysis might be slightly limited. However, in this study, most of the enrolled patients were followed up for at least 3 years, and there were enough endpoint events for the stratification of the risks of adverse cardiovascular events. Furthermore, all of the enrolled NIDCM patients had a severe cardiac dysfunction and met the primary prevention criteria for an ICD implantation. Additionally, for this specific group of patients, the normal reference range of thyroid hormone levels might not be suitable. Second, thyroid hormone levels could change over time, which may attenuate the predictive power of TSH. However, for HF patients with a thyroid function within the normal range, very few patients would like to receive a second evaluation during the course of HF therapy. In this study, we revealed that a seemingly "normal" thyroid function may not always represent a better prognosis, especially for VA events. We recommended that thyroid function should be routinely re-evaluated for NIDCM patients with a severe LV dysfunction.

In conclusion, for NIDCM patients with severely impaired LV function and euthyroid function, serum TSH levels in Q4 (>2.67 mlU/ L) may provide help for the stratification of the risk of VA events.

\section{ACKNOWLEDGMENTS}

Grants: This study was supported by National Key R\&D Program of China with grant 2017 YFC1307800.

\section{Conflicts of interest}

The authors declare no potential conflict of interests.

\section{ORCID}

Yan Yao (D) https://orcid.org/0000-0003-0670-5338 Xiaohan Fan (D) https://orcid.org/0000-0001-7661-0436

\section{REFERENCES}

1. Epstein AE, DiMarco JP, Ellenbogen KA, et al. ACC/AHA/HRS 2008 Guidelines for Device-Based Therapy of Cardiac Rhythm Abnormalities: a report of the American College of Cardiology/American Heart Association Task Force on Practice Guidelines (Writing Committee to Revise the ACC/AHA/NASPE 2002 Guideline Update for Implantation of Cardiac Pacemakers and Antiarrhythmia Devices) developed in collaboration with the American Association for Thoracic Surgery and Society of Thoracic Surgeons. J Am Coll Cardiol. 2008;51:e1-e62.

2. Hua W, Niu H, Fan $X$, et al. Preventive effectiveness of implantable cardioverter defibrillator in reducing sudden cardiac death in the
Chinese population: a multicenter trial of ICD therapy versus non-ICD therapy. J Cardiovasc Electrophysiol. 2012;23(Suppl 1):S5-S9.

3. Klein I. Thyroid and the heart: the intimacy is strained. Thyroid. 2011; 21:469-470.

4. Pearce EN, Yang Q, Benjamin EJ, Aragam J, Vasan RS. Thyroid function and left ventricular structure and function in the Framingham Heart Study. Thyroid. 2010;20:369-373.

5. Chaker L, van den Berg ME, Niemeijer MN, et al. Thyroid function and sudden cardiac death: a prospective population-based cohort study. Circulation. 2016;134:713-722.

6. Mitchell JE, Hellkamp AS, Mark DB, et al. Thyroid function in heart failure and impact on mortality. JACC Heart Fail. 2013;1:48-55.

7. Dickstein K, Cohen-Solal A, Filippatos G, et al. ESC Guidelines for the diagnosis and treatment of acute and chronic heart failure 2008: the Task Force for the Diagnosis and Treatment of Acute and Chronic Heart Failure 2008 of the European Society of Cardiology. Developed in collaboration with the Heart Failure Association of the ESC (HFA) and endorsed by the European Society of Intensive Care Medicine (ESICM). Eur Heart J. 2008;29:2388-2442.

8. Pedersen CT, Kay GN, Kalman J, et al. EHRA/HRS/APHRS expert consensus on ventricular arrhythmias. Heart Rhythm. 2014;11:e166-e196.

9. Mancia G, Laurent S, Agabiti-Rosei E, et al. Reappraisal of European guidelines on hypertension management: a European Society of Hypertension Task Force document. J Hypertens. 2009;27:2121-2158.

10. Standards of medical care in diabetes-2010. Diabetes Care. 2010;33(Suppl 1):S11-S61.

11. Moss AJ, Schuger C, Beck CA, et al. Reduction in inappropriate therapy and mortality through ICD programming. N Engl J Med. 2012;367: 2275-2283.

12. Biondi B. Mechanisms in endocrinology: heart failure and thyroid dysfunction. Eur J Endocrinol. 2012;167:609-618.

13. Baumgartner $\mathrm{C}$, da Costa BR, Collet $\mathrm{TH}$, et al. Thyroid function within the normal range, subclinical hypothyroidism, and the risk of atrial fibrillation. Circulation. 2017;136:2100-2116.

14. van Noord C, van der Deure WM, Sturkenboom MC, et al. High free thyroxine levels are associated with QTc prolongation in males. J Endocrinol. 2008;198:253-260.

15. Owecki M, Michalak A, Nikisch E, Sowiński J. Prolonged ventricular repolarization measured by corrected QT interval (QTc) in subclinical hyperthyroidism. Horm Metab Res. 2006;38:44-47.

16. Montanez A, Ruskin JN, Hebert PR, Lamas GA, Hennekens CH. Prolonged QTc interval and risks of total and cardiovascular mortality and sudden death in the general population: a review and qualitative overview of the prospective cohort studies. Arch Intern Med. 2004;164: 943-948.

17. Straus SM, Kors JA, De Bruin ML, et al. Prolonged QTc interval and risk of sudden cardiac death in a population of older adults. J Am Coll Cardiol. 2006;47:362-367.

18. Langen VL, Niiranen TJ, Puukka P, et al. Thyroid-stimulating hormone and risk of sudden cardiac death, total mortality and cardiovascular morbidity. Clin Endocrinol (Oxf). 2018;88:105-113.

19. Perez AC, Jhund PS, Stott DJ, et al. Thyroid-stimulating hormone and clinical outcomes: the CORONA trial (controlled rosuvastatin multinational study in heart failure). JACC Heart Fail. 2014;2:35-40.

20. Afsar B, Yilmaz MI, Siriopol D, et al. Thyroid function and cardiovascular events in chronic kidney disease patients. J Nephrol. 2017;30: 235-242.

21. Seo SM, Koh YS, Park HJ, et al. Thyroid stimulating hormone elevation as a predictor of long-term mortality in patients with acute myocardial infarction. Clin Cardiol. 2018;41:1367-1373.

22. Wang W, Guan H, Gerdes AM, lervasi G, Yang Y, Tang YD. Thyroid status, cardiac function, and mortality in patients with idiopathic dilated cardiomyopathy. J Clin Endocrinol Metab. 2015;100:32103218.

23. Nanchen D, Gussekloo J, Westendorp RG, et al.; PROSPER Group. Subclinical thyroid dysfunction and the risk of heart failure in older persons at high cardiovascular risk. J Clin Endocrinol Metab. 2012;97: 852-861.

24. Rhee CM, Curhan GC, Alexander EK, Bhan I, Brunelli SM. Subclinical hypothyroidism and survival: the effects of heart failure and race. J Clin Endocrinol Metab. 2013;98:2326-2336. 
25. Frey A, Kroiss M, Berliner D, et al. Prognostic impact of subclinical thyroid dysfunction in heart failure. Int J Cardiol. 2013;168:300-305.

26. Chaker L, Korevaar TIM, Rizopoulos D, et al. Defining optimal health range for thyroid function based on the risk of cardiovascular disease. J Clin Endocrinol Metab. 2017;102:2853-2861.

27. Bartalena L, Bogazzi F, Chiovato L, Hubalewska-Dydejczyk A, Links TP, Vanderpump M. 2018 European Thyroid Association (ETA) guidelines for the management of amiodarone-associated thyroid dysfunction. Eur Thyroid J. 2018;7:55-66.

28. Kusumoto FM, Bailey KR, Chaouki AS, et al. Systematic review for the 2017 AHA/ACC/HRS guideline for management of patients with ventricular arrhythmias and the prevention of sudden cardiac death: a report of the American College of Cardiology/American Heart Association Task Force on Clinical Practice Guidelines and the Heart Rhythm Society. J Am Coll Cardiol. 2018;72:1563-1676.

29. Fan $X$, Hua W, Xu Y, et al. Incidence and predictors of sudden cardiac death in patients with reduced left ventricular ejection fraction after myocardial infarction in an era of revascularisation. Heart. 2014;100:1242-1249.

30. Shiga T, Hagiwara N, Ogawa $H$, et al. Sudden cardiac death and left ventricular ejection fraction during long-term follow-up after acute myocardial infarction in the primary percutaneous coronary intervention era: results from the HIJAMI-II registry. Heart. 2009;95:216-220.

31. Scirica BM, Braunwald E, Belardinelli L, et al. Relationship between nonsustained ventricular tachycardia after non-ST-elevation acute coronary syndrome and sudden cardiac death: observations from the metabolic efficiency with ranolazine for less ischemia in non-STelevation acute coronary syndrome-thrombolysis in myocardial infarction 36 (MERLIN-TIMI 36) randomized controlled trial. Circulation. 2010;122:455-462.

32. Chen T, Koene R, Benditt DG, Lü F. Ventricular ectopy in patients with left ventricular dysfunction: should it be treated? J Card Fail. 2013;19:40-49.

33. Holmager P, Schmidt U, Mark $P$, et al. Long-term L-triiodothyronine (T3) treatment in stable systolic heart failure patients: a randomised, double-blind, cross-over, placebo-controlled intervention study. Clin Endocrinol (Oxf). 2015;83:931-937.

\section{SUPPORTING INFORMATION}

Additional supporting information may be found online in the Supporting Information section at the end of the article.

How to cite this article: Li X, Yao Y, Chen Z, et al. Thyroidstimulating hormone within the normal range and risk of major adverse cardiovascular events in nonischemic dilated cardiomyopathy patients with severe left ventricular dysfunction. Clin Cardiol. 2019;42:120-128. https://doi.org/10.1002/clc. $\underline{23117}$ 